\title{
ANÁLISIS DEL GOBIERNO CORPORATIVO EN LAS COOPERATIVAS DE AHORRO Y CRÉDITO DEL ECUADOR
}

\author{
ANALYSIS OF THE CORPORATE GOVERNANCE \\ IN ECUADOR'S SAVINGS AND CREDIT COOPERATIVES
}

\author{
ANÍBAL ALTAMIRANO SALAZAR \\ Correo electrónico: waaltamirano@espe.edu.ec \\ HENRY PAZMIÑO ARROYO \\ Correo electrónico: henryh.pazmino@ute.edu.ec \\ PILAR ESPINOSA OCHOA \\ Correo electrónico: epilar@ute.edu.ec \\ NELSON CERDA PRADO \\ Correo electrónico: ncerda@ute.edu.ec
}

FECHA DE RECEPCIÓN: 07/11/2016

FECHA DE ACEPTACIÓN: 16/12/2016

\begin{abstract}
RESUMEN
El artículo está dirigido a analizar la incidencia del gobierno corporativo en las cooperativas de ahorro y crédito, debido a que la gestión administrativa presenta mayores dificultades por la participación democrática de los socios en la toma de decisiones. Se busca también determinar si la existencia de varios actores genera diferentes tipos de relaciones entre los stakeholders y si su comportamiento provoca conflictos de interés que motiven la utilización de las prácticas creativas para ocultar el cometimiento de un fraude. En este contexto, la presente investigación busca establecer si la utilización de lineamientos de buen gobierno corporativo en las cooperativas permite prevenir los conflictos de interés, eliminar las prácticas creativas y minimizar riesgos. Para tal fin se realizó una revisión bibliográfica, que consistió en la búsqueda, organización y análisis de varios artículos científicos y de revisión relacionados con el tema, con la finalidad de construir el marco teórico y conceptual. Luego se revisó en las páginas web de las cooperativas de ahorro y crédito del Ecuador que cooperativas publican información relacionada con el gobierno corporativo y la gestión de riesgos. Se seleccionaron tres cooperativas, dos en funcionamiento y una en proceso de liquidación, para posteriormente analizar el grado de cumplimiento de las prácticas de gobierno corporativo.
\end{abstract}

Palabras clave: Gobierno Corporativo, Stakeholders, Cooperativas de Ahorro y Crédito. 


\begin{abstract}
This article aims to analyze the impact of corporate governance in the credit union, taking in account the difficulties the democratic participation of partners in decision-making presents to the administrative management. It also tries to determinate if the existence of several actors generates different types of relations between stakeholders and if their behavior causes conflicts of interest that motivate the use of creative practices to conceal the commission of a fraud. In this context, the present investigation seeks to establish whether the use of guidelines for good corporate governance in cooperatives can prevent conflicts of interest, eliminate creative practices and minimize risks. For this purpose, a literature review was done, which consisted in the searching, organization and analysis of several scientific articles and reviews related to the subject, in order to build the theoretical and conceptual framework. Then, the credit union of Ecuador websites were checked, in order to stablish which cooperatives publish information about corporate governance and risk management. Three cooperatives, two in operation and one in liquidation were selected, to later analyze the degree of compliance with corporate governance practices.
\end{abstract}

Keywords: Corporate Governance, Stakeholders, Credit Union.

\section{Introducción}

Existen diversas formas de organización empresarial, según los fines que persigan, del origen del capital y de la permisibilidad de la normativa legal en los distintos países. En las organizaciones donde prima el capital la junta de accionistas está conformada por un grupo de poder que invierten sus recursos con el único objetivo de obtener la maximización de sus utilidades. Además, en las organizaciones que pertenecen a la economía popular y solidaria la asamblea general está conformada por todos los socios sin importar el monto de capital aportado y su propiedad se encuentra dispersa entre los asociados.

En este contexto, la gestión en las cooperativas presenta mayores dificultades debido a la participación democrática de los socios en el gobierno de la misma y en la toma de decisiones, por cuanto pueden participar en las asambleas generales, elegir y ser elegidos miembros de los Consejos de Administración y Vigilancia u ocupar cargos representativos (Puentes, R., Velasco, M. y Vilar, J., 2009). Estos rasgos otorgan características especiales a la relación de agencia que se desarrolla en las cooperativas, propiciando el aparecimiento de nuevos actores.

Álvarez, (2000) señala a tres actores como integrantes de una cooperativa, los propietarios que son todos los socios, los gestores que pueden ser o no socios y los trabajadores que también pueden ser o no socios de la cooperativa. Estos actores pueden generar tres tipos de relaciones, la primera entre propietarios y gestores profesionales, típica relación de agencia. La segunda entre los socios como propietarios y los socios como gestores, denominada "especial relación de agencia", muy común en las cooperativas. Por último la relación entre la gestión y los trabajadores asalariados, denominada relación de empleo, independientemente que los gestores y los trabajadores sean socios o no.

$\mathrm{Al}$ existir muchos actores que generan varios tipos de relaciones nacen diversos tipos de interés en los stakeholders, que generan conflictos e incentivan a la utilización de prácticas creativas que distorsionan la información. La diferencia de intereses nace por tres razones fundamentales, información imperfecta, comportamiento de los agentes y sobre todo por la asimetría de la información entre las partes (Charreaux, 1987). En una cooperativa el aspecto más importante es el comportamiento de los agentes que pueden tener distintos intereses que van desde la concepción de no perder el control de la administración hasta el ocultamiento de un fraude.

Las prácticas creativas utilizadas para cambiar la información a través de la manipulación de las cifras financieras consideradas como estrate- 
gias o fraudes, son utilizadas en las cooperativas para generar una posición sólida y rentable de las mismas; es decir, los administradores buscan formar una matriz de opinión positiva frente a sus stakeholders y en especial si esta matriz tiene un efecto significativo en su valor patrimonial. Nace entonces el verdadero problema, que radica en el cumplimiento de los objetivos sin importar el cómo, generando inestabilidad e inseguridad en las operaciones, pudiendo llegar incluso a provocar el cierre de la cooperativa.

Para prevenir la utilización de las prácticas creativas, a nivel mundial se han publicado varios códigos de buen gobierno corporativo, los mismos que cuentan con carácter de obligatoriedad. En España por ejemplo se publicó el Código Olivencia en 1998, el Código Aldama en el año 2003 y el Código unificado de buen gobierno corporativo en el año 2006. En América Latina en el año 2004 el Banco de Desarrollo de América Latina, CAF, con la colaboración del IAAG, consultora con mayor experiencia especializada en gobiernos cooperativos en Latinoamérica, publicó los lineamientos para un buen gobierno corporativo actualizados en el ańo 2012. La cooperativa de las Américas ACI emitió la evaluación integral del buen gobierno cooperativo que evalúa el cumplimiento de los principios cooperativos.

El buen gobierno corporativo busca establecer los medios a través de los cuales se puede prevenir y corregir un posible conflicto de intereses entre las partes interesadas (Crespi, R. y Gispert, C., 1999). Por esta razón, la investigación busca establecer si la utilización de lineamientos de buen gobierno corporativo en las cooperativas permite prevenir los conflictos de interés, eliminar las prácticas creativas y minimizar riesgos.

\section{Metodología}

En la investigación se realizó una revisión bibliográfica que consistió en la búsqueda, organización y análisis de varios artículos científicos y de revisión relacionados con el tema. Se determinaron similitudes, concordancias y contradicciones entre los autores para argumentar la situación iden- tificada, construyendo de esta manera el marco teórico y conceptual.

Posteriormente, se revisaron en las páginas web de las cooperativas de ahorro y crédito del Ecuador, las que publican información relacionada con el Gobierno Corporativo y la gestión de riesgos. Se seleccionaron dos cooperativas, la coperativa "Andalucía" que ha implementado los lineamientos emitidos por el Banco de Desarrollo de América Latina y la cooperativa " 29 de octubre" que posee la certificación ACI de las Américas de buen gobierno cooperativo. Además, se analizaron los informes de supervisión y control realizados por la Superintendencia de Economía Popular y Solidaria y los informes de auditoría practicados a la cooperativa "Coopera", que se encuentra en liquidación.

Una vez seleccionadas tres cooperativas, dos de ellas en funcionamiento y una en proceso de liquidación se procedió a medir el grado de cumplimiento de las prácticas de Gobierno Corporativo. Para el efecto se utilizaron los lineamientos para un código de gobierno corporativo emitidos por el Banco de Desarrollo de América Latina, CAF, por cuanto evalúan cinco aspectos importantes para una buena gestión. Dichos aspectos se encuentran relacionados con los socios, la asamblea general, el directorio, la arquitectura de control y la transparencia de la información. Al final se establecieron las consecuencias que podrían originar el incumplimiento de los lineamientos del Gobierno Corporativo.

\section{Marco teórico del gobierno corporativo}

El gobierno corporativo es una temática que se encuentra inmersa en las corrientes institucionalistas y las neoinstitucionalistas. Según la corriente institucionalista las organizaciones actúan como reglas abstractas e independientes de los individuos que ayudan al logro de los objetivos, guiando al mercado (Reis Mourao, 2007). En cambio, en la corriente neoinstitucionalista las organizaciones toman relevancia y se convierten en la parte central del intercambio y de la toma 
de decisiones individual y grupal. Como se observa en las dos teorías el denominador común son las organizaciones, por esta razón es necesario analizar las teorías económicas de la organización. Entre las más relevantes se citan a la teoría de los costos de transacción, teoría de los derechos de la propiedad y la teoría de la agencia, que explican el funcionamiento de la organización y el papel del gobierno corporativo.

\section{Teoría de los Costos de Transacción}

La teoría de los costos de transacción busca identificar las características y las razones que hacen que una transacción sea problemática y costosa, con el objetivo de hacerla más eficiente (Salgado, 2003). Entendiéndose a una transacción al intercambio de bienes y servicios en el mercado o a través de un contrato, el mismo que significa una promesa de desempeño futuro. Este tipo de intercambios, donde se establece una promesa de desempeño futuro son el objeto principal de la Teoría de los Costos de Transacción (Alchian y Woodward, 1988).

Coase (1996) citado en (Bohórquez, 2011), afirma que si los costos de transacción son iguales o superiores a los costos de mercado, la empresa no tiene sentido. Para Williamson (1985) las estructuras de gobernación constituyen una gama que va del mercado a la empresa y dentro de ella los costos de transacción a que estructura de gobernación pueden pertenecer. Para el efecto la teoría de costos de transacción establece tres formas principales de gobierno: mercado, híbridos y jerarquía (Williamson O., 1991).

Los autores García, Lara y Taboada (2004) citando a Williamson (1999) manifiestan que los costos de transacción consideran en su análisis al riesgo inherente ${ }^{1}$ de las transacciones. Entre los riesgos señalan a la contratación en desequilibrio, dependencia bilateral, riesgos de medición, capacidad de respuesta, abuso estratégico, y los frágiles derechos de propiedad (Williamson O., 1999). Este tipo de riesgos en un ambiente estable carecería de interés eliminando los conflictos de contratación y la incertidumbre, mejorando la relación de mercado y organización interna. En este momento es cuando el gobierno juega un papel importante por cuanto al administrar de manera adecuada el riesgo inherente la empresa asegura la base del funcionamiento de la misma.

\section{Teoría de los Derechos de Propiedad}

El autor Demsetz (1996), establece que la importancia de los derechos de propiedad deriva del hecho que ayudan a formarse las expectativas en las relaciones con otros. Dichas expectativas se encuentran basadas en leyes, hábitos y costumbres de la sociedad, que permiten que un propietario tenga el consentimiento de sus pares para actuar de manera determinada. En cambio Rialp y Salas (2002) señalan que la propiedad es relevante en cuanto otorga derechos residuales de decisión que permiten resolver las diferencias en una transacción. Ambos autores concuerdan en que los derechos de propiedad pueden beneficiar o perjudicar a la misma persona y a terceros y el modo como lo hacen lo especifican los mismos derechos de propiedad.

La teoría establece tres tipos de propiedad, en primer lugar la propiedad conjunta es aquella en la cual las personas que participan en la transacción tienen poder de veto en los activos no humanos. En cambio en la propiedad separada, los activos no humanos que son parte de la transacción, se reparten entre todas las personas que intervienen en la misma. Por último cuando todos los activos no humanos que son parte de la transacción pertenecen a una sola persona se denominan propiedad única (Grossman, S. y Hart, O., 1986); (Hart, O. y Moore, J., 1990); (Hart, 1995).

\section{Teoría de la Agencia}

La teoría de la agencia es el marco conceptual que analiza las formas de organización económica, mediante la noción de agencia su relación y los costos que en ella se incurren (Coriat Benjamin,

1 Es aquel riesgo que se encentra inmerso en la actividad, que es parte o propio de ella. 
Weinstein Olivier, 2011). Se puede definir a la relación de agencia como el aspecto contractual entre dos partes, en la cual el principal contrata al agente para que administre sus recursos asignándole poder de decisión (Jensen, M.C. y Meckling, W.H., 1976). En este contexto la teoría de la agencia analiza los problemas que pueden surgir debido a la diferencia de intereses entre las dos partes, principal y agente. Los intereses pueden divergir por tres razones fundamentales, cuando existe información imperfecta, comportamiento de los agentes y sobre todo por la asimetría de la información entre las partes (Charreaux, 1987).

Salas Fumas (1987), manifiesta que la escasa información del principal, confiere al agente una gran discreción para perseguir objetivos propios que no siempre redundan en el mayor beneficio para el principal. Esto demuestra que la separación de la propiedad y el control son el origen de todos los problemas de la relación de la agencia, y para evitar esta disparidad se hace necesario el aparecimiento de los incentivos. Dichos incentivos permiten disminuir el nivel de riesgo que recaen sobre el principal, por cuanto con los incentivos intenta acercar o igualar los intereses. El agente en cambio asume el riesgo de tomar decisiones en un ambiente complejo, decisiones que permitirían disminuir la desviación del agente respecto a lo establecido en el contrato (Álvarez Pérez, Ma Belen; Arbesú López, Pilar; Fé Canto, Celia, 2000).

\section{Marco conceptual del gobierno corporativo}

\section{Gobierno Corporativo desde el enfoque Shareholders}

Para Shleifer y Vishny (1997) el gobierno corporativo son los medios que garantizan que las personas que invierten su dinero reciban un retorno adecuado de sus inversiones. Desde este punto de vista económico es necesario señalar que es aquí donde se configura el problema de la agencia, que consiste básicamente en la diferencia de intereses entre el principal y el agente. La existencia de la incertidumbre el riesgo y la asimetría de la información hacen que el principal no tenga el control sobre las decisiones del agente que actúa bajo sus propios intereses. Entonces nacen dos probabilidades: que el agente actúe como lo desea el principal o que el agente sea incompetente para el cargo, situaciones que impedirían que el inversionista obtenga el retorno deseado de sus inversiones.

En este sentido Crespi y Gispert (1999) coadyuban al señalar que el gobierno corporativo son mecanismos que previenen conflictos de intereses entre los acionistas y directivos. La Porta Lopez de Silanes y Shleifer y Vishny (2000), complementan este enfoque al describir como los inversores fuertes deben proteger sus derechos de propiedad contra los intereses internos. En este contexto, el gobierno corporativo busca establecer lineamientos para que las empresas sean administradas por sus directivos creando valor en beneficio de los accionistas (Ustáriz, 2004). Eisenhardt (1989) y Nilakant y Hayagreeva (1994) manifiestan que el enfoque de la teoría de la agencia (enfoque shareholders) se direcciona al esfuerzo individual dejando a un lado el trabajo en equipo.

\section{Gobierno Corporativo desde el enfoque stakeholders}

La Organización para la Cooperación y el Desarrollo Económico (OCDE) en el ańo 1999 aprobó los Principios de gobierno corporativo, que posteriormente fueron revisados en el año 2002. Dichos principios sin ser vinculantes buscan que los países miembros y no miembros de la OCDE perfeccionen sus marcos legales, institucionales y reglamentarios relacionados con el Gobierno Corporativo. En este enfoque los principios centran su atención en las empresas que cotizan en bolsa, dejando abierta la posibilidad que puede ser utilizado para todo tipo de empresas. Básicamente los principios son seis directrices que se relacionan con el marco jurídico y regulatorio, la actuación del Estado, tratamiento de los accionistas, relaciones con partes interesadas, transparencia y responsabilidades de los directorios (OCDE, 2004). 
La OCDE en su enfoque incluye a las partes interesadas, permitiendo que se defina al Gobierno Corporativo desde otro punto de vista. Para el efecto Ganga y Vera (2008) señalan que el gobierno corporativo es el conjunto de prácticas, reglas, normas y procesos apropiados que guían el comportamiento entre la administración de la empresa y los agentes económicos que tengan algún interés. Esta filosofía corporativa permite medir y evaluar los riesgos, con la finalidad garantizar el uso eficiente de los recursos y brindar confianza a una gama amplia de partes interesadas.

Para autores como Rodríguez (2002) y Rivero (2005), el gobierno corporativo es amplio y multidisciplinario que permite desarrollar la actividad empresarial con principios de transparencia y responsabilidad. Desde esta perspectiva el gobierno corporativo cuida los intereses de todas las partes interesadas, es decir accionistas, socios, directivos, empleados, acreedores, clientes, etcétera. Como se puede observar, la nueva preocupación de las empresas no es solamente la maximización de las utilidades y la valoración de sus activos, sino todos sus componentes y la comunidad en la que desarrollan sus actividades.

\section{El gobierno corporativo en las cooperativas de ahorro y crédito del Ecuador}

En el Ecuador, las cooperativas de ahorro y crédito observan lo dispuesto en la Ley orgánica de Economía Popular y Solidaria, art. 21, dicha ley define a las cooperativas de la siguiente manera:

"...como sociedades de personas que se han unido en forma voluntaria para satisfacer sus necesidades económicas, sociales y culturales en común, mediante una empresa de propiedad conjunta y de gestión democrática, con personalidad jurídica de derecho privado e interés social. Las cooperativas, en su actividad y relaciones, se sujetarán a los principios establecidos en esta Ley y a los valores y principios universales del cooperativismo y a las prácticas de Buen Gobierno Corporativo.”

En esta definición se establece que las cooperativas deben sujetarse a las prácticas de buen gobierno corporativo, y que en ellas están inmersos varios actores denominados partes interesadas. Por lo tanto, es claro que el enfoque de gobierno corporativo que debería predominar en este tipo de organizaciones es el enfoque stakeholders. Por cuanto las cooperativas no persiguen la maximización del beneficio como objetivo fin, sino que promueven objetivos con intereses sociales, en búsqueda de la satisfacción de todos los grupos de interés.

En el catastro de la Superintendencia de Economía Popular y Solidaria existen 952 cooperativas de ahorro y crédito, clasificadas en cinco segmentos, según el monto de sus activos. Al segmento No. 1, objeto de esta investigación, pertenecen 26 cooperativas cuyos activos totales de manera individual superan ochenta millones de dólares. Por el monto de sus activos, tamaño y la cantidad de socios que las conforman, estas cooperativas deberían poseer y publicar los resultados de la aplicación de los códigos, lineamientos y prácticas de buen gobierno corporativo.

Sin embargo, luego de haber revisado la información publicada en los últimos tres años, por las cooperativas que pertenecen al segmento No. 1, se obtuvieron los siguientes resultados: 
Tabla 1. Cooperativas Que Pertenecen al Segmento No.1 QUE PUBLICAN INFORMES O RESULTADOS DE GOBIERNO CORPORATIVO

\begin{tabular}{|c|c|c|c|c|}
\hline No. & Razón Social & 2013 & 2014 & 2015 \\
\hline 1 & $\begin{array}{l}\text { Cooperativa de Ahorro y Crédito Juventud } \\
\text { Ecuatoriana Progresista Ltda. }\end{array}$ & & & \\
\hline 2 & Cooperativa de Ahorro y Crédito Jardín Azuayo Ltda. & & & \\
\hline 3 & Cooperativa de Ahorro y Crédito 29 de Octubre Ltda. & $\mathrm{x}$ & $\mathrm{x}$ & $\mathrm{x}$ \\
\hline 4 & Cooperativa de Ahorro y Crédito Cooprogreso Ltda. & $\mathrm{x}$ & $\mathrm{x}$ & \\
\hline 5 & $\begin{array}{l}\text { Cooperativa de Ahorro y Crédito Vicentina } \\
\text { Manuel Esteban Godoy Ortega Ltda. }\end{array}$ & & & \\
\hline 6 & Cooperativa de Ahorro y Crédito Riobamba Ltda. & & & \\
\hline 7 & Cooperativa de Ahorro y Crédito Oscus Ltda. & & & \\
\hline 8 & Cooperativa de Ahorro y Crédito San Francisco Ltda. & & & \\
\hline 9 & $\begin{array}{l}\text { Cooperativa de Ahorro y Crédito } \\
\text { de la Pequeńa Empresa de Cotopaxi Ltda. }\end{array}$ & & $\mathrm{x}$ & \\
\hline 10 & Cooperativa de Ahorro y Crédito Andalucía Ltda. & $\mathrm{x}$ & $\mathrm{x}$ & $\mathrm{x}$ \\
\hline 11 & Cooperativa de Ahorro y Crédito Mushuc Runa Ltda. & & & \\
\hline 12 & Cooperativa de Ahorro y Crédito El Sagrario Ltda. & & & \\
\hline 13 & Cooperativa de Ahorro y Crédito 23 de Julio Ltda. & $\mathrm{x}$ & $\mathrm{x}$ & \\
\hline 14 & Cooperativa de Ahorro y Crédito Atuntaqui Ltda. & & & \\
\hline 15 & Cooperativa de Ahorro y Crédito Alianza del Valle Ltda. & & & \\
\hline 16 & $\begin{array}{l}\text { Cooperativa de Ahorro y Crédito } \\
\text { Cámara de Comercio de Ambato Ltda. }\end{array}$ & & $\mathrm{x}$ & \\
\hline 17 & Cooperativa de Ahorro y Crédito Santa Rosa Ltda. & & & \\
\hline 18 & Cooperativa de Ahorro y Crédito Pablo Muñoz Vega Ltda. & & & \\
\hline 19 & $\begin{array}{l}\text { Cooperativa de Ahorro y Crédito de la } \\
\text { Pequeña Empresa Biblián Ltda. }\end{array}$ & & & \\
\hline 20 & $\begin{array}{l}\text { Cooperativa de Ahorro y Crédito de los Servidores } \\
\text { Públicos del Ministerio de Educación }\end{array}$ & & $\mathrm{x}$ & \\
\hline 21 & Cooperativa de Ahorro y Crédito Policía Nacional Ltda. & $\mathrm{x}$ & $\mathrm{x}$ & \\
\hline 22 & Cooperativa de Ahorro y Crédito Pilahuin Tío Ltda. & & & \\
\hline 23 & Cooperativa de Ahorro y Crédito Tulcán Ltda. & & & \\
\hline 24 & Cooperativa de Ahorro y Crédito San José Ltda. & & & \\
\hline 25 & Caja Central Financoop & & $\mathrm{x}$ & \\
\hline 26 & $\begin{array}{l}\text { Cooperativa de Ahorro y Crédito } \\
\text { de la Pequeńa Empresa de Pastaza Ltda. }\end{array}$ & $\mathrm{x}$ & & \\
\hline
\end{tabular}


De las cooperativas pertenecientes a este segmento únicamente el $7.69 \%$ ha presentado de manera consecutiva los informes o resultados de gobierno corporativo en los tres períodos analizados. El 30,77\% presenta de manera parcial, es decir publican uno y hasta dos ańos, el resto de las cooperativas que representan el $61,54 \%$ no publican los informes respectivos.

Figura1. CoOperativas QUE PUbliCAN MEMORIAS

E INFormes de Gobierno CoOperativo EN LA PÁGINA WEB

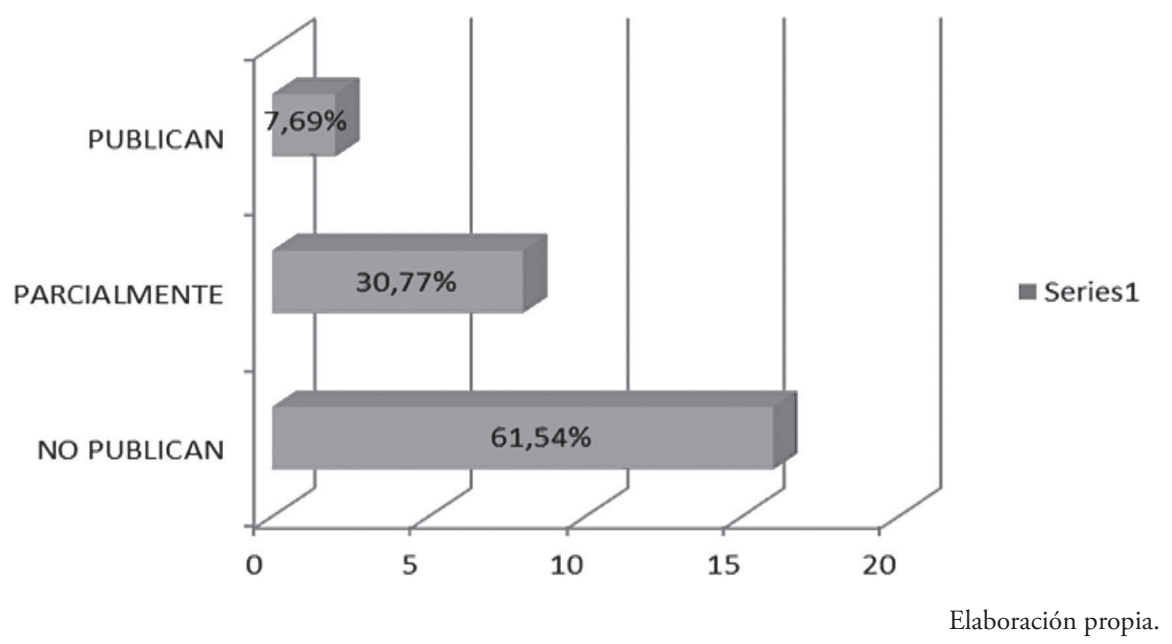

\section{Resultados}

Después de verificar el grado de cumplimiento de los lineamientos emitidos por el Banco de Desarrollo de América Latina, CAF, en las cooperativas seleccionadas, en relación con la información publicada en las páginas web y en sus informes, se obtuvieron los siguientes resultados:

Tabla 2. Metodología Banco de Desarrollo de América latina, CAF

\begin{tabular}{l|c|c|c|c}
\hline \multicolumn{1}{c}{ Lineamiento } & Puntaje óptimo & COAC Andalucía & $\begin{array}{c}\text { COAC } \\
\text { 29 de Octubre }\end{array}$ & COAC Coopera \\
\hline $\begin{array}{l}\text { Derecho y trato } \\
\text { equitativo de socios }\end{array}$ & 16 & 13 & 12 & 4 \\
\hline Asamblea General de socios & 25 & 22 & 23 & 6 \\
\hline Directorio & 56 & 49 & 45 & 9 \\
\hline Arquitectura de control & 27 & 25 & 24 & 4 \\
\hline $\begin{array}{l}\text { Transparencia de información } \\
\text { financiera y no financiera }\end{array}$ & 9 & 8 & 8 & 4 \\
\hline Total & $\mathbf{1 3 3}$ & $\mathbf{1 1 7}$ & $\mathbf{1 1 2}$ & $\mathbf{2 7}$ \\
\hline Porcentaje & $\mathbf{1 0 0 , 0 0 \%}$ & $\mathbf{8 7 , 9 7 \%}$ & $\mathbf{8 4 , 2 1 \%}$ & $\mathbf{2 0 , 3 0 \%}$ \\
\hline & & & & Elaboración propia. \\
\hline
\end{tabular}


La cooperativa Andalucía obtiene el grado de cumplimiento equivalente al $87,97 \%$, por cuanto es la única cooperativa de este segmento que publica información que da cuenta que aplica los lineamientos emitidos por el Banco de Desarrollo de América Latina. En segundo lugar se ubicó la cooperativa "29 de Octubre" que alcanzó el $84,21 \%$, no publica que haya implementado los lineamientos del banco; sin embargo, ha obtenido una certificación de buen gobierno cooperativo emitida por la ACI. Y como resultado de la evaluación de la cooperativa "Coopera" en liquidación se obtuvo un grado de cumplimiento del $20,30 \%$. El lineamiento que mayor representación tiene den- tro de la calificación total está relacionado con el Directorio y representa el $42,10 \%$. El análisis efectuado determina que las cooperativas "Andalucía" y "29 de octubre" tienen un grado de cumplimiento del $87,50 \%$ y $80,36 \%$ respectivamente. Entre las razones principales esta la dinámica del directorio mediante la utilización de planes de trabajo, reuniones periódicas, inexistencia de operaciones vinculadas, entre otras. La cooperativa Coopera obtuvo el 16,07\%, debido a la inexistencia de reglamentos, falta de requisitos para ser miembro de los consejos, remuneraciones excesivas y lo más importante existencia de operaciones vinculadas por montos significativos.

\section{Figura 2. Grado de Cumplimiento Lineamiento Directorio}

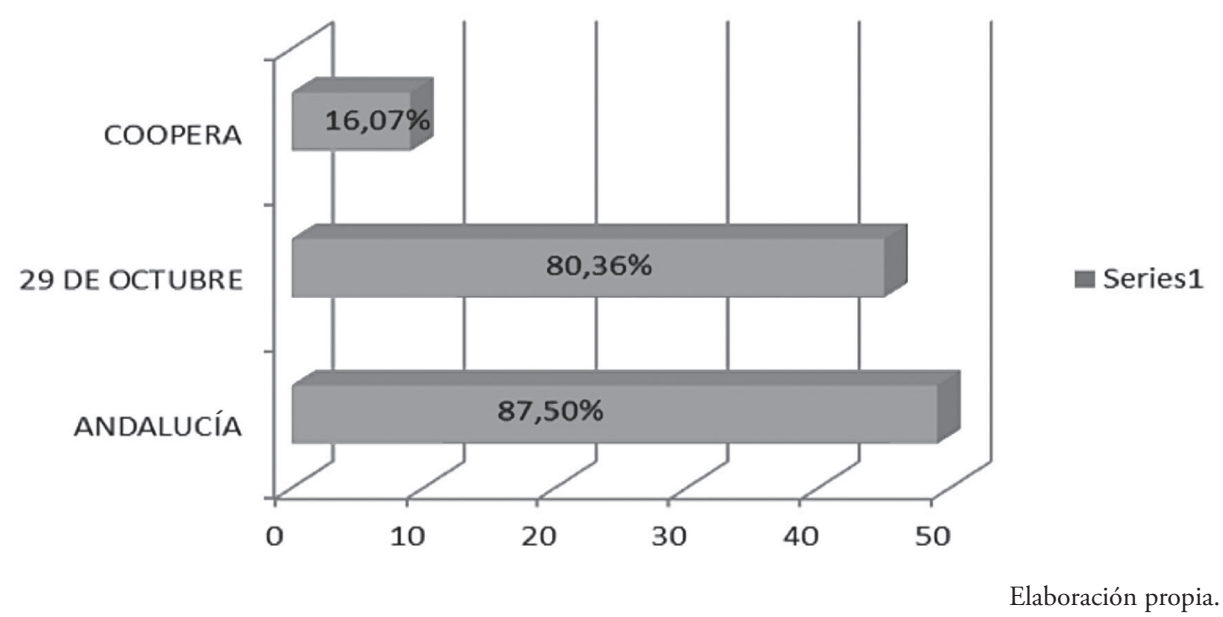

En segundo lugar están los lineamientos relacionados con la Arquitectura de Control, los mimos que están conformados por el control interno, las labores de auditoría y sobre todo la gestión de riesgos. Así mismo las dos cooperativas que han implementado prácticas de Gobierno Corporativo obtienen resultados de 24 y 25 puntos, tomando en cuenta que el puntaje óptimo es de 27 puntos. Esta situación concuerda con la información presentada por las cooperativas en sus informes de riesgos, por cuanto las dos organizaciones poseen indicadores de riesgos dentro de rangos razonables y calificaciones positivas. Los resultados demuestran que las cooperativas selec- cionadas si han establecido sistemas de control con la finalidad de minimizar sus riesgos.

Situación que no sucedió con la cooperativa "Coopera", por cuanto su bajo puntaje alcanzado demuestra las razones por las cuales se produjo su cierre y posterior liquidación. La cooperativa no poseía un sistema de control interno adecuado, por cuanto se utilizaron prácticas creativas para cambiar la realidad de la empresa. La finalidad fue el cometimiento de una serie de ilícitos, desde operaciones vinculadas con empresas ficticias en el exterior, hasta la utilización de las cuentas bancarias para el lavado de activos. 
Figura3. Grado de Cumplimiento Lineamiento Arquitectura de Control

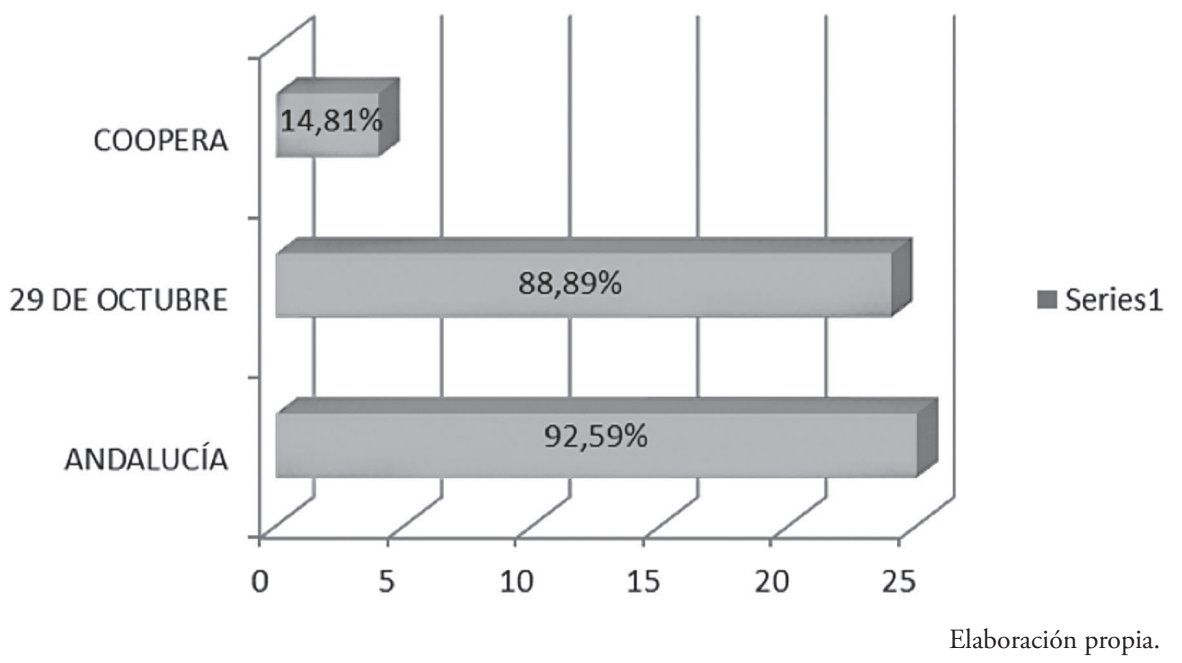

\section{Conclusiones y discusión}

La participación democrática de los socios en el gobierno de una cooperativa, ocasiona que el gobierno corporativo tenga características especiales, por cuanto existen nuevos actores y varios tipos de relaciones. Los socios que son propietarios de la cooperativa, pueden también ser gestores $u$ ocupar cargos en los mandos medios y hasta operativos, según su conocimiento y especialización. Esta situación otorga rasgos especiales a la relación de agencia, por cuanto existen diversos tipos de interés al existir varios stakeholders.

La diferencia de intereses ocasiona conflictos que afectan a la gestión de una cooperativa, por cuanto sus administradores buscan cambiar la realidad de la información mediante la utilización de prácticas creativas. Realizadas con la finalidad de mantener el control de la administración de la cooperativa u ocultar un fraude. Esta última, es la razón más predominante, por cuanto las cooperativas se han convertido en focos de atención para poder hacer lícito el dinero obtenido de manera ilícita, lavado de activos, entre otros delitos.

En la investigación se observó la existencia de un caso de lavado de activos, ocultado con la utilización de prácticas contables creativas que ocasionaron el cierre de la misma, fraude que fue cometido por cuanto los controles internos establecidos por la cooperativa no garantizaban seguridad y razonabilidad de la información. El Directorio no estaba estructurado de manera adecuada, existía operaciones vinculadas con empresas ficticias en el exterior.

En cambio, en las cooperativas que implementaron lineamientos de buen gobierno corporativo, se observa que poseen indicadores aceptables y calificaciones positivas. Dentro de sí se han establecido verdaderos sistemas de control, sus directorios son conformados por miembros idóneos con alta capacitación y todos sus procesos se encuentran debidamente reglamentados.

En conclusión, las prácticas de buen gobierno corporativo minimizan los conflictos de interés, por la tanto disminuye la posibilidad de utilización de prácticas contables creativas que cambien la realidad de una cooperativa, en beneficio de los administradores. Una cooperativa que aplique gobierno corporativo sin importar la metodología utilizada va a obtener indicadores positivos que le permitirán minimizar riesgos que hagan más eficiente su administración.

El gobierno corporativo es un tema nuevo, conforme se demostró en esta investigación, por lo menos no hay información publicada que de- 
muestre que ha sido implementado en las cooperativas de ahorro y crédito del Ecuador. En tal virtud se recomienda que se seleccione una muestra de varias cooperativas y se implementen lineamientos de gobierno corporativo, los mismos que deberán ser evaluados de manera periódica e ir analizando los resultados obtenidos. Con la finalidad de comparar los resultados frente a las cooperativas que no han sido seleccionadas y proponer una metodología que sea aplicable de manera obligatoria con el apoyo de los organismos de control.

\section{Referencias bibliográficas}

- Alchian y Woodward. (1988). The Firm is Dead; Long Live the Firm: A review of Oliver E. Williamsons. The Economic institution of capitalism. Journal of economic literature(26), 65-79.

- Álvarez Pérez, Ma Belén; Arbesú López, Pilar; Fé Canto, Celia. (2000). Las cooperativasen el marco de la teoría de la agencia. Ciriec España, Revista de Economia Pública, Social y Cooperativa(34), 169-188.

- Bohórquez, L. E. (2011). Gobierno corporativo y control interno: algunas limitaciones y desafios. Economía Gestión y Desarrollo(12), 39-57.

- Charreaux, G. (1987). La théorie positive de l'agence: une synthese de la litterature. De novvelles theories pour gérer I'Entreprise.

- Coriat Benjamin, Weinstein Olivier. (2011). Nuevas teorias de la empresa una revisión crítica. Buenos Aires: Lenguaje claro.

- Crespi, R. y Gispert, C. (1999). Implications for the governance of Spanish companies. II foro de finanzas.

- Demsetz, H. (1996). Una revisión de la teoria de la empresa. En o. Williamson,\& S. Winter, la naturaleza de la empresa: origenes y desarrollo. Fondo de Cultura económica., 220-247.

- Eisenhardt, K. (1989). Agency theory: An assessment and review. Academy of Management Review(14), 57-74.
- Ganga Contreras Francisco Anibal, Vera Garnica José Ricardo. (2008). El Gobierno coorporativo: consideraciones y cimientos teóricos. Cuadernos de administración, 21(35), 93-126.

- García Garnica, Alejandro; Lara Rivero, Arturo; Taboada Ibarra, Eunice. (2004). La coordinación "híbrida" desde las perspectivas de Williamson y de Nooteboom. Análisis Económico, XIX(40), 102-117.

- Grossman, S. y Hart, O. (1986). the costs and benefits of ownership: a theory of vertical and lateral integration. Journal of political econo$\operatorname{mic}(94), 691-719$.

- Hart, O. (1995). Firms, Contracts, and Financial Structure. Clarendon Press Oxford.

- Hart, O. y Moore, J. (1990). Property rights and the nature of the Firm. Journal of Political Economy(98), 119-1158.

- Jensen, M.C. y Meckling, W.H. (1976). Theory of the firm: Managenial behavior, agency cost, and ownership structure. Journal of Financial Economics, 3, 305-360.

- La Porta López de Silanes y Shleifer y Vishny. (2000). La protección del inversor y gobierno corporativo. Revista de Economía Financiera, 58, 3-27.

- Nilakant, V. y Hayagreeva, R. (1994). Agency theory and uncertainty in organizations: an evaluation. Organization studies(15), 649-672. 
- OCDE, O. p. (2004). Principios de Gobierno Corporativo de la OCDE. París: Lerko Print S.A.

- Puentes, R., Velasco, M. y Vilar, J. (2009). El buen gobierno corporativo en las sociedades cooperativas. Revista de Estudios Cooperati$\operatorname{vos}(98), 118-140$.

- Reis Mourao, P. (2007). El institucionalismo norteamericano: origenes y presente. Revista de Economia Institucional(9), 315-325.

- Rialp, Josep; Salas, Vicente. (2002). La Colaboración empresarialdesde la teoría de los derechos de propiedad. Investigaciones Económicas, $X X I V, 113-144$.

- Rivero, P. (2005). Responsabilidad social y Gobierno Corporativo: Información y transparencia. Revista asturiana de economía RAE(34), 9-29.

- Rodríguez, J. (2002). Teoría de los partícipies y ciudadania empresarial: Una perspectiva pluralista del gobierno de las companias. Ekonomiaz, 74-109.

- Salas Fumas, V. (1987). Economía de la Empresa. Barcelona: Ariel.
- Salgado, E. (2003). Teoría de costos de transacción una breve reseña. Cuadernos Administrativos Bogotá, 61-78.

- Shleifer, A; Vishny R. (1997). A Survey of Corporate Governance. Journal of Finance(52), 737-783.

- Ustáriz, L. (2004). Corporate Governance: evolución y reto frente al riesgo operativo en el nuevo acuerdo de Basilea. (C. N. Corporativo, Ed.) Boletin de Corporate Governance(53).

- Williamson, O. (1985). Las Instituciones económicas del capitalismo. México.

- Williamson, O. (1991). Comparative economic organization. The analysis of discrete structural alternatives. Administrative Sciencie Quarterly, 36, 269-296.

- Williamson, O. (1999). Nueva vista al realismo legal: la perspectiva del derecho, la economía y la organización”, en Carroll, G. y Teece, D., Empresas, mercados y jerarquías. La perspectiva económica de los costos de transacción. Oxford University Press. 